\title{
Identification of a diacylglycerol acyltransferase gene involved in accumulation of triacylglycerol in Mycobacterium tuberculosis under stress
}

\author{
Correspondence \\ Pappachan E. Kolattukudy \\ pk@mail.ucf.edu
}

Received 13 March 2006

Revised 10 May 2006

Accepted 11 May 2006

\author{
Tatiana D. Sirakova, $\uparrow$ Vinod S. Dubey, $\uparrow$ Chirajyoti Deb, Jaiyanth Daniel, \\ Tatiana A. Korotkova, Bassam Abomoelak and Pappachan E. Kolattukudy
} Biomolecular Science Center, and Department of Molecular Biology and Microbiology, Burnett
College of Biomedical Sciences, University of Central Florida, Orlando, FL 32816-2364, USA

\begin{abstract}
Mycobacterium tuberculosis under stress stores triacylglycerol (TG). There are 15 genes in $M$. tuberculosis that belong to a novel family of TG synthase genes (tgs), but it is not known which of them is responsible for this accumulation of TG. In this paper, it is reported that M. tuberculosis H37Rv accumulated TG under acidic, static or hypoxic growth conditions, or upon treatment with NO, whereas TG accumulation was drastically reduced in the tgs 1 (Rv3130c) disrupted mutant. Complementation with $\operatorname{tgs} 1$ restored this $\mathrm{TG}$ accumulation. $\mathrm{C}_{26}$ was a major fatty acid in this TG, indicating that the TGS1 gene product uses $\mathrm{C}_{26}$ fatty acid, which is known to be produced by the mycobacterial fatty acid synthase. TGS1 expressed in Escherichia coli preferred $\mathrm{C}_{26: 0}$-CoA for TG synthesis. If TG storage is needed for the long-term survival of $M$. tuberculosis under dormant conditions, the tgs 1 product could be a suitable target for antilatency drugs.
\end{abstract}

\section{INTRODUCTION}

Tuberculosis (TB), one of the biggest killers among the infectious diseases, poses a major public health problem mostly in the developing world with 8 million new cases and 2 million deaths a year (World Health Organization, 2005; Zhang, 2004). Mycobacterium tuberculosis, the causative agent of $\mathrm{TB}$, is inhaled by people as an aerosol emanating from active TB patients. Usually, only a small fraction of the people $(\sim 5 \%)$ thus infected develop active TB, while the rest carry a latent infection for the rest of their lives until reactivation of the pathogen occurs upon weakening of the immune system. Thus, AIDS causes reactivation of the latent tubercle bacillus leading to rapid bacillary growth and dissemination, resulting in the development of active TB (Cosma et al., 2003; Zahrt, 2003). The ability of the pathogen to go into a latent/dormant non-replicating state, with very low metabolic activity and phenotypic resistance to all of the currently available antituberculosis drugs (Zhang, 2004; Zahrt, 2003), makes TB eradication an extremely difficult challenge.

The metabolic and physiological state of the dormant pathogen that allows it to survive inside the host for decades remains unclear. There is strong evidence that fatty acids are the energy source used by the pathogen for its long-term survival during the persistence phase of infection (Gomez \& McKinney, 2004; Russell, 2003). In M. tuberculosis, two

Abbreviations: ICL, isocitrate lyase; TB, tuberculosis; TG, triacylglycerol. †These authors contributed equally to this work. genes, icl1 and icl2, encode isocitrate lyase (ICL), a key metabolic enzyme involved in fatty acid utilization. It has recently been shown that deletion of both icll and icl2, but not deletion of either one alone, results in complete impairment of intracellular replication in macrophages and rapid elimination from mouse lungs (Munoz-Elias \& McKinney, 2005). Chemical inhibition of both ICL1 and ICL2 also blocks the growth of $M$. tuberculosis on fatty acids and in macrophages. However, the source of the fatty acids remains unknown (Munoz-Elias \& McKinney, 2005; Russell, 2003). Recently it has been shown that $M$. tuberculosis can synthesize and store triacylglycerol (TG) as an energy reserve under hypoxia and NO treatment, stress conditions among others that lead to a dormancy-like state in culture (Daniel et al., 2004). Intracellular TG inclusion bodies found in pathogenic $M$. tuberculosis obtained from organ lesions (Garton et al., 2002), and decreased host-cell TG levels induced by growth of Mycobacterium bovis BCG in macrophages (Jackson et al., 1989), suggest that TG is used as an energy source for intracellular growth of the pathogen.

Fifteen members of a novel class of diacylglycerol acyltransferase genes designated tgs (triacylglycerol synthase) have been identified (Daniel et al., 2004), based on homology to a gene found in Acinetobacter calcoaceticus (Kalscheuer \& Steinbuchel, 2003). Which, if any, of these genes encodes the enzyme(s) actually involved in TG synthesis is not known. In this report, we show that tgs 1 deficiency abolishes TG accumulation under several stress conditions, and that complementation with tgs 1 restores TG accumulation. We also show that TGS1 expressed in 
Escherichia coli preferentially uses $\mathrm{C}_{26}$ fatty acyl-CoA for TG synthesis, and that $\mathrm{C}_{26}$ fatty acid, which is known to be produced by the fatty acid synthase of $M$. tuberculosis (Kikuchi et al., 1992), is a major long-chain fatty acid in the TG accumulated under stress conditions.

\section{METHODS}

Bacterial strains, growth media and chemicals. $M$. tuberculosis H37Rv (ATCC 25618), the tgs 1 mutant and the complemented strain were grown in Middlebrook 7H9 (Difco), Dubos-Tweenalbumin medium broth (Difco) and Sauton medium, as previously described (Daniel et al., 2004). E. coli DH5 $\alpha$ and HB101 strains (Life Technologies) were used for cloning and propagation of plasmids and phasmids. For selection of transformants, E. coli was grown in Luria-Bertani (LB) broth or on agar. Mycobacterium smegmatis $\mathrm{mc}^{2} 155$ was grown in liquid LB medium with $0.5 \%$ Tween 80 for competent cell preparation, and in Middlebrook 7H9 broth with $0.05 \%$ Tween 80 for transduction. When required, antibiotics were added to the culture media at the following concentrations: ampicillin, $100 \mu \mathrm{g} \mathrm{ml}^{-1}$ for E. coli; hygromycin B, $150 \mu \mathrm{g} \mathrm{ml}^{-1}$ for E. coli or $75 \mu \mathrm{g} \mathrm{ml}^{-1}$ for M. tuberculosis; and kanamycin, $50 \mu \mathrm{g} \mathrm{ml}^{-1}$ for $E$. coli or $20 \mu \mathrm{g} \mathrm{ml}^{-1}$ for $M$. tuberculosis. The NO donor (spermine NONOate) and its reference compound spermine tetrahydrochloride were purchased from Alexis Corporation. Other chemicals and antibiotics were from Sigma and Fisher Scientific. DNA restriction and modifying enzymes were obtained from New England Biolabs. AcylCoA substrates were purchased from American Radiolabelled Chemicals.

Growth conditions for $\boldsymbol{M}$. tuberculosis. We subjected M. tuberculosis to several in vitro stress conditions, such as hypoxia, NO treatment, low $\mathrm{pH}$ and static growth. For hypoxia and NO treatment, experiments were done as previously described (Daniel et al., 2004). For hypoxia experiments, the initial $\mathrm{OD}_{600}$ of the starting culture was 0.06 , and, at each time point, an equal volume of cell culture $(\sim 200 \mathrm{ml})$ from each strain was harvested, and the cells were used for lipid extraction. At each time point, an equal proportion of lipids from each strain was used for (TLC) separation for TG analysis. Resistance to isoniazid $\left(0 \cdot 4 \mu \mathrm{g} \mathrm{ml}^{-1}\right)$ and sensitivity to metronidazole $\left(12 \mu \mathrm{g} \mathrm{ml}^{-1}\right)$ of hypoxic cultures were also tested (Daniel et al., 2004). The culture conditions for the NO treatment experiment were the same as those previously described (Daniel et al., 2004), except that the cells from each strain were collected after $6 \mathrm{~h}$ of NO treatment, and used for RNA isolation and TG analysis. For low-pH growth, $M$. tuberculosis cells were initially grown in Middlebrook $7 \mathrm{H} 9$ media to $\mathrm{OD}_{600} 0 \cdot 6-0 \cdot 8$, and the cells were washed and suspended in Middlebrook $7 \mathrm{H} 9$ media, $\mathrm{pH} 5 \cdot 0$, and grown for 3 weeks in a roller bottle at $37^{\circ} \mathrm{C}$. For growth under static conditions, $M$. tuberculosis cells were grown for 2 weeks in Middlebrook $7 \mathrm{H} 9$ media to $\mathrm{OD}_{600} 1 \cdot 6$, and then the culture was left to stand for 1 week at $37^{\circ} \mathrm{C}$. Cells collected at different time periods were used for RNA isolation and TG analysis.

Generation of $\boldsymbol{t g s} \mathbf{1}$-disrupted mutant of $\boldsymbol{M}$. tuberculosis. The general strategy used for gene disruption with the specialized transducing phage system was similar to that used previously by Bardarov et al. (2002) and Sirakova et al. (2001). tgs1 was disrupted by allelic exchange via specialized transduction using conditionally replicating mycobacteriophage phAE87. The disrupted copy of the gene was constructed by PCR amplification of the $5^{\prime}$ - and $3^{\prime}$-flanking regions of the gene using $M$. tuberculosis DNA as a template, and primer pairs introducing BspHI and PstI sites, and $\mathrm{XhoI}$ and $B s p \mathrm{HI}$ sites, at the $5^{\prime}$ and $3^{\prime}$ ends of the products, respectively (Table 1 ). Both products were then sequentially inserted on either side of the hygromycin-resistance gene ( $h y g)$ cloned in vector pUC19. The $5^{\prime}$-flanking region consisted of the first $10 \mathrm{bp}$ of tgs 1 and the $784 \mathrm{bp}$ sequence upstream of the gene. The 3 '-flanking region contained the last $122 \mathrm{bp}$ of the tgsl ORF and the $667 \mathrm{bp}$ downstream sequence. The disrupted copy of the gene was released by $B s p H I$ digestion, and cloned into pYUB572, which contains a bacteriophage lambda cos site and a PacI site. The recombinant cosmid was digested with PacI, ligated into the PacI site of phAE87, and the resultant recombinant phage was used to transduce wild-type $M$. tuberculosis. Individual hygromycin-resistant colonies were analysed by PCR and Southern blot analysis to identify clones in which allelic exchange had occurred at the $\operatorname{tgs} 1$ locus. Several $\operatorname{tgs} 1$ mutants were screened by PCR analysis

Table 1. Primers used for making the tgs 1 disruption construct in $M$. tuberculosis

\begin{tabular}{|c|c|c|}
\hline Primer pair & Primer & Sequence \\
\hline \multicolumn{3}{|c|}{ Primer pairs used to generate $5^{\prime}$ and $3^{\prime}$ flanks of tgs 1 genes } \\
\hline \multirow[t]{2}{*}{$5^{\prime}$ flank } & A & $5^{\prime}$-tcatgatcttggcgatctccagc- $3^{\prime}$ \\
\hline & B & $5^{\prime}$-ctgcagggtgattcatggtcagc- $3^{\prime}$ \\
\hline \multirow[t]{2}{*}{$3^{\prime}$ flank } & $\mathrm{C}$ & $5^{\prime}$-ctcgagacgtggtagcagatgcc- $3^{\prime}$ \\
\hline & $\mathrm{D}$ & $5^{\prime}$-tcatgatacttccegcactgccc- $3^{\prime}$ \\
\hline \multicolumn{3}{|c|}{ Primer pairs inside the deleted segment } \\
\hline & $\Delta \mathrm{F}$ & $5^{\prime}$-ttatcgtcgctcgctcaacgcc- $3^{\prime}$ \\
\hline & $\Delta \mathrm{R}$ & $5^{\prime}$-tttccgtaatcgcggcaagcgc- $3^{\prime}$ \\
\hline \multicolumn{3}{|c|}{ Primer pairs used for genomic flanks } \\
\hline \multirow[t]{2}{*}{$5^{\prime}$ flank } & $\mathrm{E}$ & $5^{\prime}$-cggcattgatcggtgcccaaccc- $3^{\prime}$ \\
\hline & $\mathrm{H} 1$ & $5^{\prime}$-tgaggcgatggtggtgtcgatgct- $3^{\prime}$ \\
\hline \multirow[t]{2}{*}{$3^{\prime}$ flank } & $\mathrm{H} 2$ & $5^{\prime}$-ggaactggcgcagttcctctgggg- $3^{\prime}$ \\
\hline & $\mathrm{F}$ & $5^{\prime}$-tcacgcccaaactccaacacaccg-3' \\
\hline \multicolumn{3}{|c|}{ Primer pairs used for RT-PCR } \\
\hline \multirow[t]{2}{*}{$\operatorname{tgs} 1$} & $\mathrm{~F}$ & $5^{\prime}$-tgggtcgtcgacatgggtggcgag- $3^{\prime}$ \\
\hline & $\mathrm{R}$ & $5^{\prime}$-cgtgctaagtcccgccgcgtc- $3^{\prime}$ \\
\hline \multirow[t]{2}{*}{$23 \mathrm{~S}$ rRNA } & $\mathrm{F}$ & $5^{\prime}$-gtggcgtgttctggacccgaagcg-3' \\
\hline & $\mathrm{R}$ & $5^{\prime}$-gtccatcgactacgcctgtcggcc- $3^{\prime}$ \\
\hline
\end{tabular}


using specific primers located inside the deleted fragment of the gene $(\Delta \mathrm{F}$ and $\Delta \mathrm{R})$, as shown in Table 1 . The allelic exchange by double crossover was confirmed with two sets of primers, each representing a hyg primer (primers $\mathrm{H} 1$ and $\mathrm{H} 2$ ) and a primer (primers $\mathrm{E}$ and $\mathrm{F}$ ) in the mycobacterial genome outside the gene sequence used for making the disruption construct (Fig. 1A).

Complementation of $\boldsymbol{M}$. tuberculosis tgs 1 mutant. For complementation experiments, the tgs 1 coding sequence was amplified from genomic M. tuberculosis H37Rv DNA using Pfu Turbo Hotstart DNA Polymerase (Stratagene) and primers that introduced a KpnI site at both ends. The PCR product was cloned into pCR-Blunt IITOPO (Invitrogen). After sequencing to confirm the absence of any mutations, the insert was excised from this vector by $K p n I$ digestion, and subcloned into KpnI-digested replicative shuttle vector pMIP12 for expression under the pBlaF* promoter (LeDantec et al., 1991). The construct was electroporated into the tgs1-disrupted mutant, and transformants were selected on $7 \mathrm{H} 11$ plates with both kanamycin and hygromycin. Isolated colonies from the plates were checked for the expression of tgs 1 by RT-PCR using the primers shown in Table 1. RNA isolation and RT-PCR analysis were done as described previously (Daniel et al., 2004).

Genomic DNA isolation and Southern blotting. M. tuberculosis H37Rv genomic DNA was isolated by the guanidine thiocyanate (GTC) method using a solution of GTC, Tris/ $\mathrm{HCl}$ and sarcosyl (Derbyshire \& Bardarov, 2000). DNA samples were digested with EcoRI and EcoRI/BamHI restriction enzymes, subjected to $1 \%$ agarose gel electrophoresis, transferred to nylon membranes (Nytran Plus; Schleicher and Schuell), and hybridized with $\left[\alpha-{ }^{32} \mathrm{P}\right] \mathrm{dCTP}-$ labelled probes using the random prime labelling system Rediprime II (Amersham Pharmacia).
TG analysis. M. tuberculosis wild-type, the tgs 1 mutant and the complemented strain of $t g s 1$ cells were subjected to the desired stress at defined experimental time points, recovered by centrifugation, autoclaved and subjected to lipid extraction in chloroform/methanol $(2: 1, \mathrm{v} / \mathrm{v})$ (Sirakova et al., 2001). In a separate set of hypoxia experiments, aliquots of the culture of $M$. tuberculosis wild-type and the tgs 1 mutant, taken at various time points, were incubated with $50 \mu \mathrm{M}$ oleic acid in $0.5 \%$ BSA solution, and after $6 \mathrm{~h}$ incubation, lipids were extracted. At each defined time point, under various stress conditions, an equal proportion of the total extracted lipids ( 5-10 mg) from each strain was subjected to Silica-G TLC for TG analysis, using n-hexane/diethylether $(9: 1, \mathrm{v} / \mathrm{v})$ as a solvent system. Dichromate/sulfuric acid charring of TLC plates was used to detect lipids. The charred TLC plates were also scanned for quantification of TG accumulation using the AlphaImager 2200 Gel Doc system (Alpha Innotech). Fatty acid methyl esters were prepared from TG by transesterification with $14 \% \mathrm{BF}_{3}$ in methanol, and analysed by (GC) using the flame-ionization detector response; the GC conditions for fatty acid methyl esters were the same as those previously described (Sirakova et al., 2001). All stress growth experiments were repeated at least three times, and similar patterns were seen in all cases. Since details such as cell density were not absolutely identical in all repetitions, we present the mean values and results from a typical experiment.

Enzyme activity of TGS1 expressed in E. coli. TGS1 was expressed in E. coli grown in Dual Media (Zymo Research) for $12 \mathrm{~h}$ at $37^{\circ} \mathrm{C}$, and total cell lysates were assayed for TGS activity. The assays were performed in $0 \cdot 1 \mathrm{M}$ citrate/phosphate buffer, $\mathrm{pH} 6.5$, containing $1 \mathrm{mg} \mathrm{BSA}, 10 \mathrm{mM} \mathrm{MgCl} 2,14 \cdot 5 \mu \mathrm{M}\left[{ }^{14} \mathrm{C}\right]$ diolein (specific activity $55 \mathrm{Ci} \mathrm{mol}^{-1}, 2 \cdot 04 \mathrm{TBq} \mathrm{mol}^{-1}$, American Radiolabelled Chemicals), $500 \mu \mathrm{M}$ diolein, indicated concentrations of oleoyl

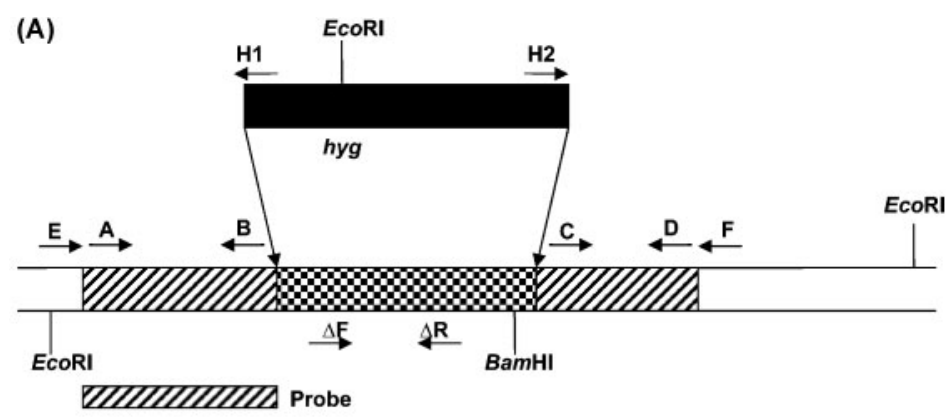

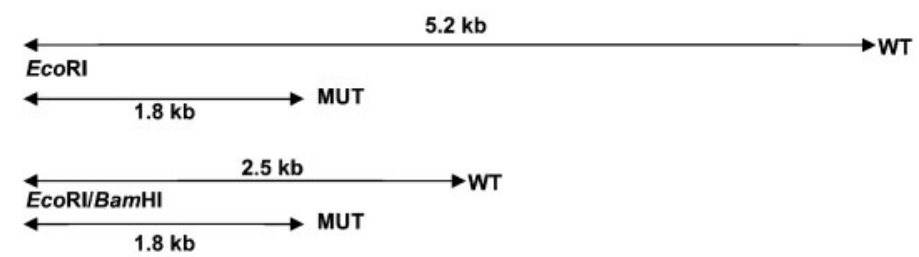

(B)

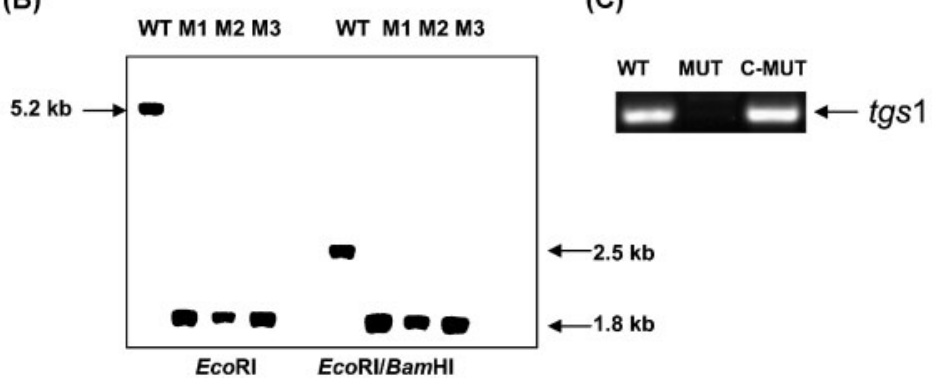

Fig. 1. Generation of a tgs 1 mutant of $M$. tuberculosis. (A) Schematic representation of the disruption construct for tgs 1 . Hatched and checkered segments represent the regions used to make the disruption construct. The checkered segment was replaced with the hyg gene cassette (black box). Primer pairs $E / H 1, H 2 / F$ and $\Delta F / \Delta R$ were used for $\mathrm{PCR}$ analysis of homologous recombination as described in the text. (B) Southern blot hybridization of the wild-type (WT) and three mutant clones (M1, M2 and M3). (C) RT-PCR analysis showing the tgs1 transcript in the WT and tgs 1 complemented strains (C-MUT), but not in the tgs 1 mutant (MUT). 
$\left(\mathrm{C}_{18: 1}\right)$-CoA or hexacosanoyl $\left(\mathrm{C}_{26: 0}\right)$-CoA and $100-200 \mu \mathrm{g}$ protein in a total volume of $250 \mu \mathrm{l}$ for $2 \mathrm{~h}$ at $37^{\circ} \mathrm{C}$. Following the assay, the reaction mixture was extracted and the TG product was quantified as described previously (Daniel et al., 2004).

\section{RESULTS}

\section{Disruption of tgs 1 in M. tuberculosis and complementation of the disrupted mutant}

On the basis of the enzymic activities of expressed tgs gene products and the level of their induction as the pathogen was subjected to hypoxia and NO stress conditions, tgs 1 appeared to have the greatest potential for involvement in TG synthesis (Daniel et al., 2004). To test this possibility, we generated a tgs1-disrupted mutant of M. tuberculosis. tgs1 was disrupted by allelic exchange via specialized transduction using conditionally replicating mycobacteriophage phAE87 (Bardarov et al., 2002). To prepare the tgs1 disruption construct, a 1260 bp tgs1 ORF, out of the total $1392 \mathrm{bp}$, was replaced with the hygromycin-resistance gene, and was used as the substrate for allelic exchange by double crossover (Fig. 1A). PCR screening of the hygromycinresistant transductants with a set of primers $(\Delta \mathrm{F}$ and $\Delta \mathrm{R})$ specific for the deleted segment identified several mutants that failed to amplify the expected $662 \mathrm{bp}$ fragment (data not shown). Disruption of tgs 1 by homologous recombination was confirmed by further PCR analysis of the flanking regions (primer pairs $\mathrm{E} / \mathrm{H} 1$ and $\mathrm{H} 2 / \mathrm{F}$ ), which yielded the expected-size products. Southern blot analysis of $M$. tuberculosis wild-type and three tgs 1 mutants is shown in Fig. 1(B). Genomic DNA from M. tuberculosis digested with EcoRI and BamHI/EcoRI showed a $5 \cdot 2 \mathrm{~kb}$ and a $2.5 \mathrm{~kb}$ hybridization band, respectively, when the $5^{\prime}$-flanking region of the construct was used as the probe. DNA from the mutants, under the same conditions, showed a $1.7 \mathrm{~kb}$ band from both digestions, due to the presence of an EcoRI site in the hyg gene sequence and the absence of a BamHI site. RT-PCR analysis showed that the mutant did not produce any tgs 1 transcripts (Fig. 1C).

We used a replicative shuttle vector, pMIP12, to complement the disrupted mutant by expressing the tgs1 ORF under the control of the $\beta$-lactamase promoter $\left(p b l a F^{\star}\right)$ of Mycobacterium fortuitum, one of the strongest mycobacterial promoters (LeDantec et al., 1991). After electroporation of the expression construct into the tgs1-disrupted mutant, transformants were selected on $7 \mathrm{H} 11$ plates supplemented with both kanamycin and hygromycin. Isolated colonies were checked for the restored expression of tgs1. RT-PCR analysis of the complemented strain showed that tgs1 was expressed (Fig. 1C).

\section{TG analysis of $M$. tuberculosis, tgs 1 mutant and complemented mutant under hypoxia}

Previously we have shown that M. tuberculosis accumulates TG under hypoxia (Daniel et al., 2004). To test whether $t g s 1$ deficiency affected the ability to accumulate TG under such conditions, we cultured the cells under hypoxia. Under these conditions, the pathogen developed isoniazid resistance and metronidazole susceptibility by 16 days. The samples from both the wild-type and tgs 1 mutant were collected at $0,6,11$ and 16 days, and extracted lipids were separated by TLC and subjected to dichromate/sulfuric acid charring to visualize TG. The tgs 1 mutant showed no detectable level of TG at any time points under hypoxic growth conditions, whereas the wild-type started accumulating TG from day 6 and reached a maximum at day 16 (Fig. 2A). Lipids were isolated from the silica gel region corresponding to TG, and fatty acid analysis was done by GC. This analysis showed that TG from the wild-type contained all fatty acids ranging from $\mathrm{C}_{16}$ to $\mathrm{C}_{30}$, with $\mathrm{C}_{26}$ as the major fatty acid among the longer-chain fatty acids, whereas $\mathrm{C}_{26}$ fatty acid in the tgs 1 mutant was virtually absent (Fig. 3 ). To determine whether TG accumulation could be restored in the tgs 1 mutant by providing exogenous fatty acids, we added oleic acid as a BSA complex at days 6,11 and 16 to both wild-type and tgs 1 mutant cell cultures. Charring of lipids extracted from these cultures showed that the tgs 1 mutant at days 11 and 16 contained a drastically reduced level of TG compared to the wild-type (Fig. 2B). Even the trace amounts of TG

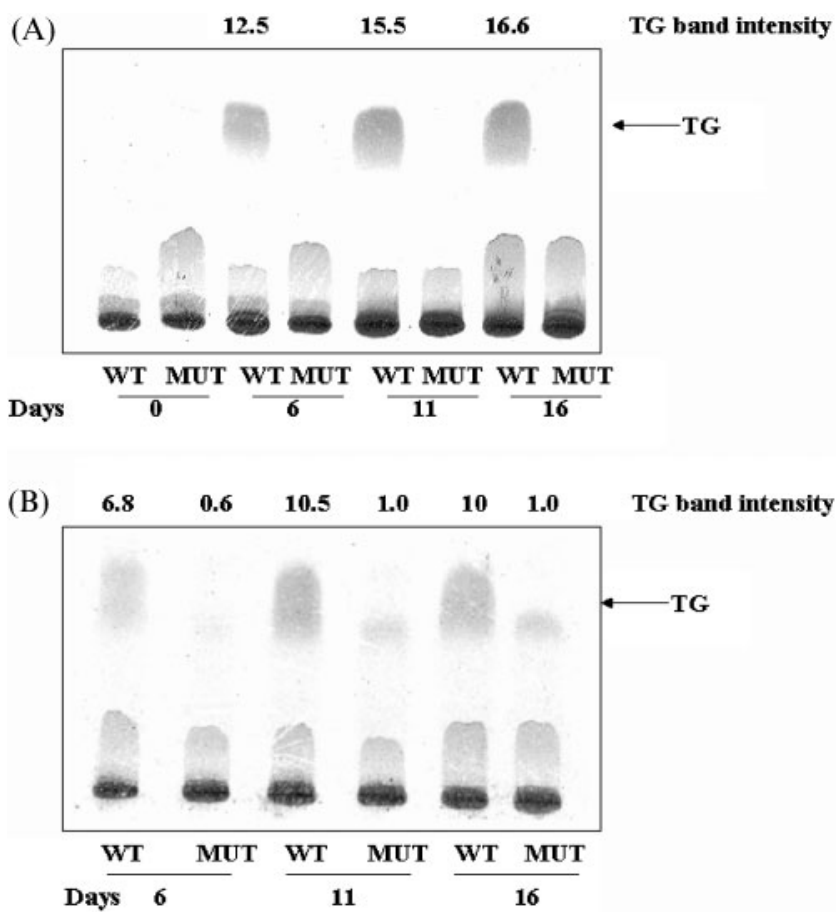

Fig. 2. Dichromate/sulfuric acid charring of lipids showing TG accumulation in $M$. tuberculosis under hypoxia. At each time point total lipids were extracted from $M$. tuberculosis WT and MUT (A), and after $6 \mathrm{~h}$ incubation with $50 \mu \mathrm{M}$ oleic acid $/ 0.5 \%$ BSA (B). In each case, the same proportion of lipids was separated by TLC using $n$-hexane/diethyl ether $(9: 1, v / v)$. The intensity of the TG band determined in arbitrary units by the Alphalmager $2200 \mathrm{Gel}$ Doc system is shown at the top of each panel. For strain abbreviations, see legend to Fig. 1. 


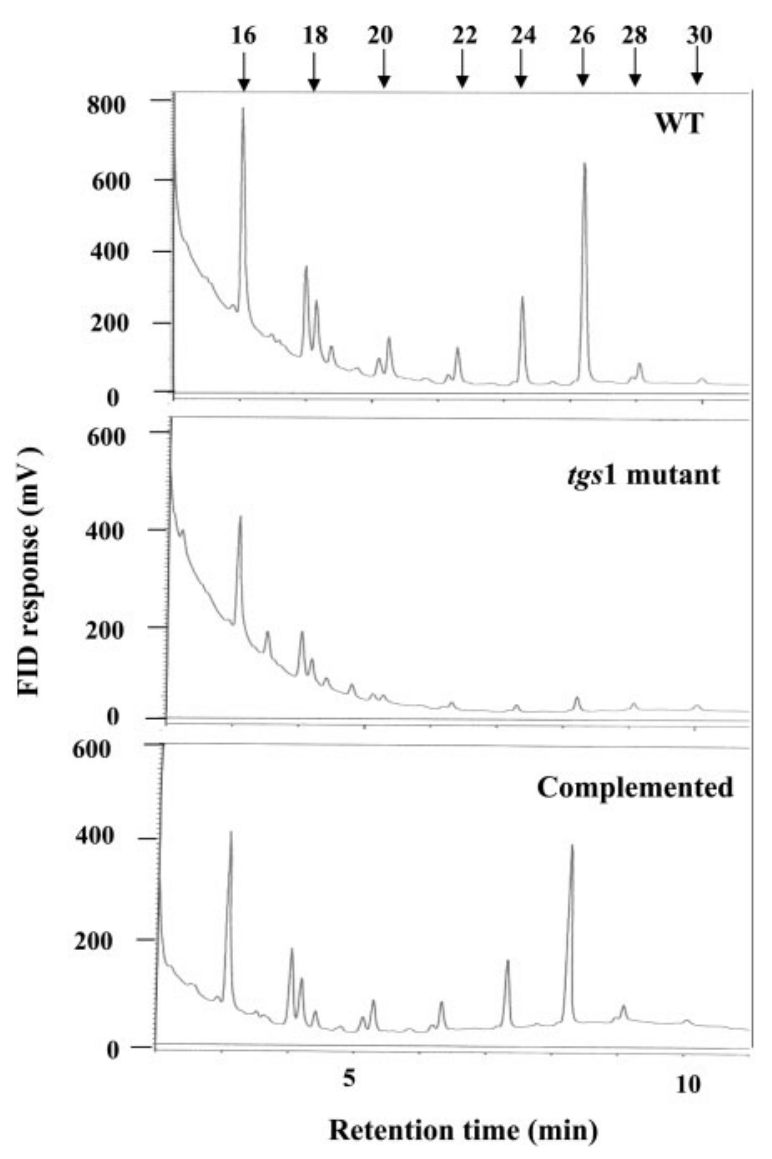

Fig. 3. $\mathrm{GC}$ analysis of fatty acids in TG from $M$. tuberculosis cells after 16 days under hypoxia. Fatty acid methyl esters were prepared from TG recovered from TLC plates from the wildtype (WT; top panel), tgs 1 mutant (middle panel) and complemented tgs 1 mutant (bottom panel). Retention times of $n$-fatty acids are indicated at the top.

present in the tgs 1 mutant contained small amounts of the fatty acids present in the wild-type $\mathrm{TG}$, but $\mathrm{C}_{26}$ was absent (data not shown). The small amount of TG produced by the oleic acid-supplemented cultures of the tgs 1 mutant contained oleic acid as a major component and some $\mathrm{C}_{16}$ $\mathrm{C}_{24}$ fatty acids in similar proportions to those found in the wild-type, but $\mathrm{C}_{26}$ was barely detectable (data not shown).

In a separate set of experiments, we also subjected the complemented strain of the $\operatorname{tgs} 1$ mutant to the same hypoxic conditions to see if it could restore TG accumulation. Cultures of the wild-type, $\operatorname{tg} s 1$ mutant and complemented $\operatorname{tgs} 1$ strain were grown under hypoxia for 16 days, the lipids extracted from the cells were separated by TLC, and the plates were subjected to dichromate/sulfuric acid charring. The results showed that the $\operatorname{tgs} 1$ mutant was unable to accumulate TG, whereas its complemented strain accumulated TG just as in the wild-type (Fig. 4). TG isolated from both the wild-type and the complemented strain showed a

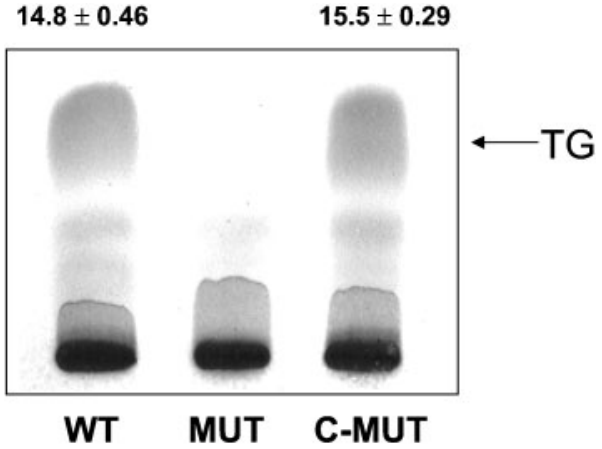

Fig. 4. TG accumulation in M. tuberculosis WT, MUT and CMUT after 16 days under hypoxia. The same proportion of lipids was separated by TLC using $n$-hexane/diethyl ether $(9: 1$, $\mathrm{v} / \mathrm{v}$ ), and lipids were visualized by dichromate/sulfuric acid charring. A charred TLC chromatogram is shown from a typical experiment, and the intensity of the TG band was determined in arbitrary units by the Alphalmager 2200 Gel Doc system. The values are given as the mean \pm SEM of three independent measurements. For strain abbreviations, see legend to Fig. 1.

similar fatty acid profile, with $\mathrm{C}_{26}$ as the major fatty acid among the longer-chain fatty acids, whereas $\mathrm{C}_{26}$ fatty acid was virtually absent in the $\operatorname{tgs} 1$ mutant (Fig. 3).

\section{Acyl chain length preference of recombinant TGS1}

The fatty acid analysis of the TG produced by the wild-type and tgs 1 mutant suggested that TGS1 may prefer $\mathrm{C}_{26}$ fatty acyl-CoA as a substrate. To test for this possibility, we expressed TGS1 in E. coli and assayed the TGS activity in the total cell lysate using both $\mathrm{C}_{18: 1^{-}}$and $\mathrm{C}_{26: 0^{-}} \mathrm{CoA}$ as substrates. Results clearly showed that TGS1 had a preference for $\mathrm{C}_{26: 0}-\mathrm{CoA}$ over $\mathrm{C}_{18: 1}$-CoA (Fig. 5). The $K_{\mathrm{m}}$ values for $\mathrm{C}_{26: 0^{-}} \mathrm{CoA}$ and $\mathrm{C}_{18: 1^{-}}$CoA were calculated to be 306 and

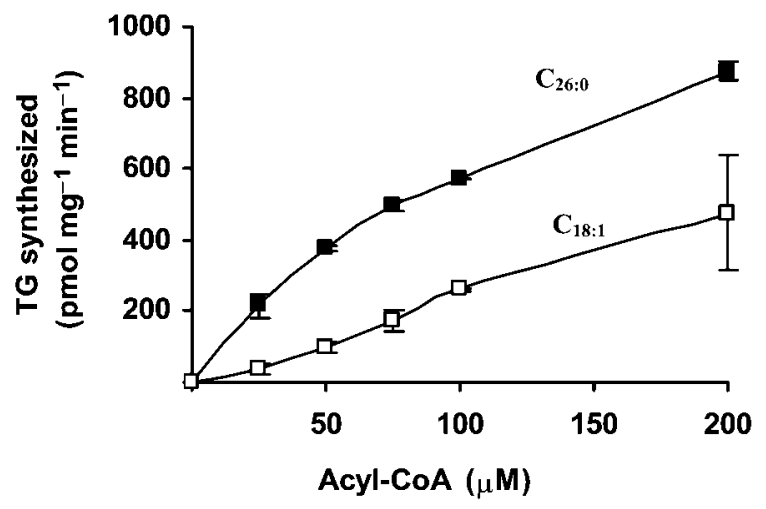

Fig. 5. Effects of acyl chain length on TG synthesis by recombinant TGS1. Total cell lysates of $E$. coli expressing TGS1 were assayed for TGS activity using $\mathrm{C}_{26: 0}-\mathrm{CoA}(\boldsymbol{\square})$ and $\mathrm{C}_{18: 1}-\mathrm{CoA}(\square)$. 
$540 \mu \mathrm{M}$, respectively, from linear double-reciprocal plots. The respective $V_{\max }$ values for $\mathrm{C}_{26: 0^{-}} \mathrm{CoA}$ and $\mathrm{C}_{18: 1}$ - CoA were calculated to be $2 \cdot 4$ and $1 \cdot 1 \mathrm{nmol} \mathrm{mg}^{-1} \mathrm{~min}^{-1}$.

\section{Role of tgs1 in TG synthesis under acidic and static growth conditions and upon NO treatment}

Besides hypoxic stress, bacilli are also believed to encounter other stress factors, such as acidic $\mathrm{pH}$ in caseous granuloma, slow growth in the static phase, and NO production inside the host macrophages (Chan et al., 2001; Florczyk et al., 2003; Nathan, 2002; Saviola et al., 2003). Therefore, we tested whether the tgs 1 mutant was able to accumulate TG under such stress conditions. $M$. tuberculosis wild-type, the tgs 1 mutant and the tgs 1 complemented strain were grown under acidic stress conditions, and TG accumulation was analysed. After 3 weeks growth in acidic medium, the wildtype accumulated TG, but it was not detected in the tgs 1 mutant, whereas the tgs 1 complemented strain accumulated a level of TG similar to that found in the wild-type (Fig. 6A). Fatty acid analysis of the TG accumulated under acidic conditions showed that the major fatty acids were $\mathrm{C}_{16}-\mathrm{C}_{28}$, with $\mathrm{C}_{26}$ as the major component among the longer-chain fatty acids. The tgs 1 mutant showed no detectable level of $\mathrm{C}_{26}$ fatty acid, even in the trace amount of TG that was found. The complemented tgs 1 mutant showed a TG fatty acid profile identical to that of the wild-type (data not shown).

tgs 1 is reported to be induced in $M$. tuberculosis grown under static conditions (Florczyk et al., 2003). To test whether TG accumulates, and to ascertain whether the tgs 1 product is involved in TG synthesis under such conditions, we tested the wild-type, tgs 1 mutant and the complemented mutant. Cell cultures grown for 2 weeks in Middlebrook 7H9 medium in a roller incubator were allowed to settle, and were further incubated for 1 week as standing cultures. Total lipids were extracted from these cultures and analysed by TLC. Dichromate/sulfuric acid charring results showed that the tgs 1 mutant lost the ability to accumulate TG. The complemented strain accumulated TG to a level similar to that observed in the wild-type (Fig. 6B). Fatty acid analysis of the TG from both the wild-type and complemented mutant strain showed a similar fatty acid profile, with $\mathrm{C}_{16}$ and $\mathrm{C}_{18}$ being the major species, along with a very low level of longer-chain fatty acids $\left(\mathrm{C}_{20}-\mathrm{C}_{26}\right)$, whereas in the tgs 1 mutant that showed the same proportion of smaller-chain fatty acids, $\mathrm{C}_{26}$ was not even detectable (data not shown).

Treatment of M. tuberculosis with NO is known to trigger induction of the hypoxia regulon, including tgs1 (Ohno et al., 2003; Voskuil et al., 2003), and to cause accumulation of TG (Daniel et al., 2004). To test for the role of tgs 1 in TG accumulation, we subjected the tgs 1 mutant and its complemented strain to NO treatment. After $6 \mathrm{~h} \mathrm{NO}$ treatment, cells were harvested for total lipid extraction. The lipids were separated by TLC, and dichromate/sulfuric acid charring of the TLC plate showed that the TG level in the
(A)

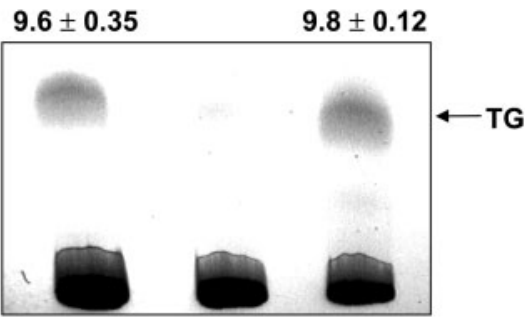

(B)

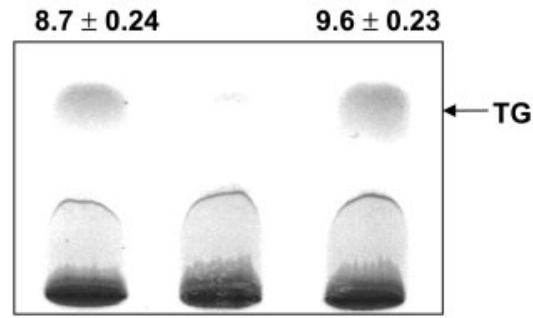

(C)

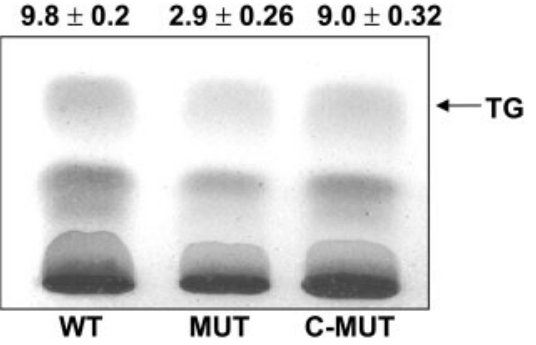

Fig. 6. Dichromate/sulfuric acid charring of lipids showing TG accumulation in $M$. tuberculosis grown under stress conditions. Total lipids extracted from WT, MUT and C-MUT under low $\mathrm{pH}$ (A) or static growth conditions (B), or with NO treatment (C) are shown. In each case, the same proportion of lipids was separated by TLC using $n$-hexane/diethyl ether $(9: 1, v / v)$. Charred TLC chromatograms are shown from a typical experiment, and the intensity of the TG band was determined in arbitrary units by the Alpha Imager 2200 Gel Doc system. The values are given as the mean \pm SEM of three independent measurements. For strain abbreviations, see legend to Fig. 1.

tgs1 mutant was much less (30\%) than that in the wild-type, whereas the complemented strain accumulated TG to a level comparable to that found in the wild-type (Fig. 6C). Fatty acid analysis of TG isolated from both the wild-type and the tgs1-complemented strain showed $\mathrm{C}_{16}-\mathrm{C}_{28}$ fatty acids, with $\mathrm{C}_{26}$ as a major component; however, in the tgs 1 mutant, the level of $\mathrm{C}_{26}$ fatty acid was drastically reduced (data not shown).

\section{RT-PCR analyses of tgs transcript levels under various stress culture conditions}

Induction levels of $15 \mathrm{tgs}$ genes were assessed by semiquantitative RT-PCR analyses of mRNA isolated from cells of the wild-type, tgs 1 mutant and tgs 1 -complemented strains grown under different stress conditions. The tgs 1 transcript was completely absent in the $t g s 1$ mutant but it was induced 
in the wild-type and tgs1-complemented strains under all the stress conditions, as expected. Transcript levels of the other tgs genes under hypoxic, static and acidic growth conditions were similar in all three strains; however, in NOtreated cells of the tgs 1 mutant, transcript levels of a few tgs genes (Rv3371, Rv1760, Rv2285 and Rv1425) were slightly higher than those in the wild-type and tgs1-complemented strains (data not shown).

\section{DISCUSSION}

In spite of the strong evidence for the use of fatty acids as the major source of energy for growth in the persistent stage (Munoz-Elias \& McKinney, 2005; Russell, 2003; Segal \& Bloch, 1956; Wayne \& Sohaskey, 2001), the source of fatty acids has not been identified. TG is widely used as a storage form of energy in many living organisms. Being a completely reduced form of substrate, TG can generate much higher levels of energy than other types of storage materials such as carbohydrates. For long-term survival with very low metabolic rates, TG is used by many organisms, including animals in hibernation, dormant oil seeds and fungal spores. We postulated that the fatty acids used during the long-term survival of M. tuberculosis in the persistent/dormant state are stored in the form of TG (Daniel et al., 2004). This hypothesis is supported by the finding that TG-containing inclusion bodies appear soon after the provision of fatty acids, and under nutrient starvation (Garton et al., 2002). Growth of M. bovis BCG in macrophages causes decreased TG levels in the host cells (Jackson et al., 1989), suggesting that TG is used as an energy source for the intracellular growth of the pathogen. These findings suggest that lipid body formation and accumulation of TG play an important role in the metabolism of pathogenic M. tuberculosis.

We have previously identified 15 genes in the M. tuberculosis genome which could potentially encode TGS; all 15 tgs genes are expressed in this pathogen in culture, and their products expressed in E. coli show TGS activity when tested with dioleoylglycerol and oleoyl-CoA as substrates (Daniel et al., 2004). Some of these tgs genes have been found to be highly induced as the pathogen enters a non-replicating state in culture, upon slow withdrawal of oxygen and upon treatment with NO (Daniel et al., 2004), two conditions known to trigger induction of some of the genes potentially involved in latency/dormancy (Ohno et al., 2003; Voskuil et al., 2003). Based on the enzymic activities of the expressed tgs gene products and the degree of their induction, tgs1 appears to have the potential to be a major contributor to TG synthesis induced by hypoxia and NO (Daniel et al., 2004). To assess the role of the tgs 1 locus in TG accumulation, tgs 1 was disrupted by allelic exchange using a phage-mediated delivery system. We subjected the pathogen to various in vitro stress conditions, and found that all of these resulted in accumulation of TG in the wild-type, but in the tgs 1 mutant, TG accumulation was drastically reduced under such conditions. To confirm that the inability of the mutant to accumulate TG was due to inactivation of the tgsl gene, and not to any secondary effects, we complemented the mutant with a copy of the tgs 1 gene expressed under the strong $p B l a F^{\star}$ promoter on the replicative plasmid pMIP12. Under similar in vitro stress conditions, where the tgs 1 mutant showed drastically reduced levels of TG accumulation, complementation of the $\operatorname{tgs} 1$ gene restored the ability of the pathogen to accumulate TG.

Oxygen limitation (hypoxic conditions) during granuloma development has been proposed as one of the main signals that can change the metabolic status of the bacilli so that they enter the dormant state (Wayne \& Sohaskey, 2001). tgs1 has been found to be part of the dormancy regulon (Park et al., 2003), and its promoter region contains a motif characteristic of the promoters of the $\alpha$-crystalline homologue protein (acr) gene family, which is consistent with the gene being upregulated under hypoxic conditions (Florczyk et al., 2003). It is noteworthy that tgs 1 shows high induction in vivo within macrophages upon IFN- $\gamma$ activation (Schnappinger et al., 2003), under both hypoxia and NO treatment (Daniel et al., 2004; Ohno et al., 2003; Park et al., 2003; Voskuil et al., 2003), and in static culture (Florczyk et al., 2003). Along with several other stress factors, such as hypoxia, nutrient deprivation, and production of $\mathrm{NO}$ and IFN- $\gamma$ by host cells (Chan et al., 2001; Nathan, 2002; Wayne \& Sohaskey, 2001), acidic $\mathrm{pH}$ is also considered to be one of the major stress factors which bacteria may encounter inside the host granuloma (Saviola et al., 2003). Previously, when $M$. tuberculosis was subjected to 15 min acidic shock, changes in tgs 1 expression were not reported (Fisher et al., 2002). However, in our experimental conditions, where M. tuberculosis was grown under acidic conditions for 3 weeks, we observed accumulation of TG in the wild-type pathogen, whereas the tgs 1 mutant did not accumulate TG, implicating an important role for tgs 1 in acidic growth conditions.

Complementation restored TG storage with similar fatty acids, with $\mathrm{C}_{26}$ as the major long-chain fatty acid com ponent. The $\mathrm{C}_{26}$ preference of TGS1, suggested by these results, was further supported by the finding that recombinant TGS1 showed significantly higher TGS activity with $\mathrm{C}_{26: 0^{-}} \mathrm{CoA}$ as a substrate than with $\mathrm{C}_{18: 1^{-}}$CoA. Since $\mathrm{C}_{26}$ fatty acid is exclusively produced by $M$. tuberculosis and not synthesized by its host, the preference of TGS1 protein for $\mathrm{C}_{26: 0^{-}}$-CoA over $\mathrm{C}_{18: 1^{-}}$-CoA implies its central role in utilizing the endogenously synthesized long-chain $\mathrm{C}_{26}$ fatty acid. $M$. tuberculosis is known to have a multifunctional fatty acid synthase that generates fatty acids with a bimodal distribution centred at $C_{16}$ and $C_{26}$ (Kikuchi et al., 1992), as previously reported for Mycobacterium phlei (Brindley et al., 1969). The present results show that $C_{26}$ is a major fatty acid in the TG stored by the pathogen under stress. When exogenous $\left[{ }^{14} \mathrm{C}\right]$ oleic acid is provided to the pathogen under stress, ${ }^{14} \mathrm{C}$ is incorporated into $\mathrm{C}_{26}$, as well as the $\mathrm{C}_{18: 1}$ found in the stored TG (Daniel et al., 2004), indicating that exogenous fatty acids are catabolized, and the $\mathrm{C}_{2}$ units generated are used for the synthesis of $\mathrm{C}_{26}$ by the fatty acid 
synthase within the pathogen. We found that $\mathrm{C}_{26}$ was absent even in the very small amount of TG that was made by the tgs1-deficient organism.

We have already demonstrated the impaired ability of the tgs 1 mutant in storing TG under in vitro stress conditions; however, it is possible that different sets of tgs genes are induced in response to the different stress factors encountered in the host by the pathogen, in order to enable the organism to synthesize TG with maximum efficiency, if TG is indeed used in vivo as the main form of energy storage in dormancy. Elevated expression of other tgs genes has also been reported in various in vitro growth conditions. Microarray analysis has shown that, under nutrient starvation conditions, Rv3734c, Rv3087 and Rv3088 are upregulated, whereas Rv3233c and Rv3234c are repressed (Betts et al., 2002). Rv3087 and Rv3088 are also upregulated after 15 min of acidic shock to M. tuberculosis (Fisher et al., 2002), and Rv3087 and Rv3371 are predicted to be required for in vivo survival in mice (Sassetti \& Rubin, 2003). It is possible that under in vivo conditions, different tgs genes are induced under the influence of the different host factors.

\section{ACKNOWLEDGEMENTS}

This work was supported in part by grants AI46582 and AI35272 from the National Institutes of Health.

\section{REFERENCES}

Bardarov, S., Bardarov, S., Jr, Pavelka, M. S., Jr, Sambandamurthy, V., Larsen, M., Tufariello, J., Chan, J., Hatfull, G. \& Jacobs, W. R., Jr (2002). Specialized transduction: an efficient method for generating marked and unmarked targeted gene disruptions in Mycobacterium tuberculosis, M. bovis BCG and M. smegmatis. Microbiology 148, 3007-3017.

Betts, J. C., Lukey, P. T., Robb, L. C., McAdam, R. A. \& Duncan, K. (2002). Evaluation of a nutrient starvation model of Mycobacterium tuberculosis persistence by gene and protein expression profiling. Mol Microbiol 43, 717-731.

Brindley, D. N., Matsumura, S. \& Bloch, K. (1969). Mycobacterium phlei fatty acid synthase - a bacterial multienzyme complex. Nature 224, 666-669.

Chan, E. D., Chan, J. \& Schluger, N. W. (2001). What is the role of nitric oxide in murine and human host defense against tuberculosis? Current knowledge. J Respir Cell Mol Biol 25, 606-612.

Cosma, C. L., Sherman, D. R. \& Ramakrishnan, L. (2003). The secret lives of the pathogenic mycobacteria. Annu Rev Microbiol 57, 641676

Daniel, J., Deb, C., Dubey, V. S., Sirakova, T. D., Abomoelak, B., Morbidoni, H. R. \& Kolattukudy, P. E. (2004). Induction of a novel class of diacylglycerol acyltransferases and triacylglycerol accumulation in Mycobacterium tuberculosis as it goes into a dormancy-like state in culture. J Bacteriol 186, 5017-5030.

Derbyshire, K. M. \& Bardarov, S. (2000). DNA transfer in mycobacteria: conjugation and transduction. In Molecular Genetics of Mycobacteria, pp. 93-107. Edited by G. F. Hatfull \& W. R. Jacobs Jr. Washington, DC: American Society for Microbiology.

Fisher, M. A., Plikaytis, B. B. \& Shinnick, T. M. (2002). Microarray analysis of the Mycobacterium tuberculosis transcriptional response to the acidic conditions found in phagosomes. J Bacteriol 184, 40254032 .

Florczyk, M. A., McCue, L. A., Purkayastha, A., Currenti, E., Wolin, M. J. \& McDonough, K. A. (2003). A family of acracr-coregulated Mycobacterium tuberculosis genes shares a common DNA motif and requires $\mathrm{Rv} 3133 \mathrm{c}$ (dosR or $\operatorname{devR}$ ) for expression. Infect Immun 71, 5332-5343.

Garton, N. J., Christensen, H., Minnikin, D. E., Adegbola, R. A. \& Barer, M. R. (2002). Intracellular lipophilic inclusions of mycobacteria in vitroin vitro and in sputum. Microbiology 148, 2951-2958.

Gomez, J. E. \& McKinney, J. D. (2004). M. tuberculosis persistence, latency, and drug tolerance. Tuberculosis 84, 29-44.

Jackson, S. K., Stark, J. M., Taylor, S. \& Harwood, J. L. (1989). Changes in phospholipids fatty acid composition and triacylglycerol content in mouse tissues after infection with bacilli CalmetteGuerin. Br J Exp Pathol 70, 435-441.

Kalscheuer, R. \& Steinbuchel, A. (2003). A novel bifunctional wax ester synthase/acyl-CoA:diacylglycerol acyltransferase mediates wax ester and triacylglycerol biosynthesis in Acinetobacter calcoaceticus ADP1. J Biol Chem 278, 8075-8082.

Kikuchi, S., Rainwater, D. L. \& Kolattukudy, P. E. (1992). Purification and characterization of an unusually large fatty acid synthase from Mycobacterium tuberculosis var. bovis BCG. Arch Biochem Biophys 295, 318-326.

LeDantec, C., Winter, N., Gicquel, B., Vincent, V. \& Picardeau, M. (1991). Genomic sequence and transcriptional analysis of a 23kilobase mycobacterial linear plasmid:evidence for horizontal transfer and identification of plasmid maintence systems. $J$ Bacteriology 183, 2157-2164.

Munoz-Elias, E. J. \& McKinney, J. D. (2005). Mycobacterium tuberculosis isocitrate lyases 1 and 2 are jointly required for in vivo growth and virulence. Nat Med 11, 638-644.

Nathan, C. (2002). Inducible nitric oxide synthase in the tuberculous human lung. Am J Respir Crit Care Med 166, 130-131.

Ohno, H., Zhu, G., Mohan, V. P., Chu, D., Kohno, S., Jacobs, W. R., Jr \& Chan, J. (2003). The effects of reactive nitrogen intermediates on gene expression in Mycobacterium tuberculosis. Cell Microbiol 5, 637-648.

Park, H. D., Guinn, K. M., Harrell, M. I., Liao, R., Voskuil, M. I., Tompa, M., Schoolnik, G. K. \& Sherman, D. R. (2003). Rv3133c/ dosR is a transcription factor that mediates the hypoxic response of Mycobacterium tuberculosis. Mol Microbiol 48, 833-843.

Russell, D. G. (2003). Phagosomes, fatty acids and tuberculosis. Nat Cell Biol 5, 776-778.

Sassetti, C. M. \& Rubin, E. J. (2003). Genetic requirements for mycobacterial survival during infection. Proc Natl Acad Sci U S A 100, 12989-12994.

Saviola, B., Woolwine, S. C. \& Bishai, W. R. (2003). Isolation of acidinducible genes of Mycobacterium tuberculosis with the use of recombinase-based in vivo expression technology. Infect Immun 71, 1379-1388.

Schnappinger, D., Ehrt, S., Voskuil, M. I. \& 8 other authors (2003). Transcriptional adaptation of Mycobacterium tuberculosis within macrophages: insights into the phagosomal environment. J Exp Med 198, 693-704.

Segal, W. \& Bloch, H. (1956). Biochemical differentiation of Mycobacterium tuberculosis grown in vivo and in vitro. $J$ Bacteriol 72, 132-141.

Sirakova, T. D., Thirumala, A. K., Dubey, V. S., Sprecher, H. \& Kolattukudy, P. E. (2001). The Mycobacterium tuberculosis pks2 gene encodes the synthase for the hepta- and octamethyl-branched fatty acids required for sulfolipid synthesis. J Biol Chem 276, 1683316839. 
Voskuil, M. I., Schnappinger, D., Visconti, K. C., Harrell, M. I., Dolganov, G. M., Sherman, D. R. \& Schoolnik, G. K. (2003). Inhibition of respiration by nitric oxide induces a Mycobacterium tuberculosis dormancy program. J Exp Med 198, 705-713.

Wayne, L. G. \& Sohaskey, C. D. (2001). Nonreplicating persistence of Mycobacterium tuberculosis. Annu Rev Microbiol 55, 139-163.
World Health Organization (2005). Global tuberculosis control. http://www.who.int/tb/publications/2005/en/index.html.

Zahrt, T. C. (2003). Molecular mechanisms regulating persistent Mycobacterium tuberculosis infection. Microbes Infect 5, 159-167.

Zhang, Y. (2004). Persistent and dormant bacilii and latent tuberculosis. Front Biosci 9, 1136-1156. 